\title{
Absolute photoionization cross sections and resonance structure of doubly ionized silicon in the region of the $2 p^{-1}$ threshold: Experiment and theory
}

\author{
J.-P. Mosnier,* M. H. Sayyad, ${ }^{\dagger}$ and E. T. Kennedy \\ National Centre for Plasma Science and Technology, School of Physical Sciences, Dublin City University, Glasnevin, Dublin 9, Ireland \\ J.-M. Bizau, ${ }^{\ddagger}$ D. Cubaynes, and F. J. Wuilleumier \\ Laboratoire d'Interaction des rayons X avec la Matière and LURE, Bat 350, Université Paris-Sud, F-91405 Orsay Cedex, France
}

J.-P. Champeaux and C. Blancard

Département de Physique Théorique et Appliquée, CEA/DAM Ile-De-France, F-91680 Bruyères-le-Châtel, France

R. Hari Varma, T. Banerjee, and P. C. Deshmukh

Department of Physics, Indian Institute of Technology_Madras, Chennai 600036, India

S. T. Manson

Department of Physics and Astronomy, Georgia State University, Atlanta, Georgia 30303, USA

(Received 14 July 2003; published 21 November 2003)

\begin{abstract}
We present the absolute photoionization cross section of doubly ionized silicon as a function of photon energy. These were obtained by merging a $\mathrm{Si}^{2+}$ ion beam generated in an electron cyclotron resonance source with monochromatized synchrotron radiation from an undulator. The photoion yield measurements were carried out in the photon energy range between $95 \mathrm{eV}$ and $170 \mathrm{eV}$, i.e., the region corresponding to the excitation followed by the ionization (threshold $\sim 133.8 \mathrm{eV}$ ) of an inner-subshell $2 p$ electron. Resonance structure due to $2 p$ excitation in the $2 p^{6} 3 s 3 p^{3} P$ metastable state was also observed with its contribution to the total cross section not exceeding 3\%. Calculation of the $2 p$ photoionization continuum cross section as a function of photon energy was carried out using the relativistic random-phase approximation (RRPA) and agreed very well with the corresponding measurements. The resonance structure in the $3 s$ cross section below the $2 p$ threshold was found to be in good agreement with the multiconfiguration atomic structure calculations of Sayyad et al. [J. Phys. B 28, 1715 (1995)], while the corresponding RRPA-RMQDT (relativistic multi-channel quantumdefect theory) calculations proved less successful.
\end{abstract}

DOI: 10.1103/PhysRevA.68.052712

\section{INTRODUCTION}

Absolute data are much needed for the critical evaluation of the modern computational codes that predict fundamental atomic properties such as photoionization cross sections or discrete oscillator strengths. The recent emergence of large theoretical efforts represented in projects such as OPACITY, OPAL, or IRON [1] testifies to this need. Furthermore, photoionization data are important for the interpretation of laboratory and astrophysical plasma phenomena as they can provide detailed information on the inverse processes of radiative and dielectronic recombination, which are key processes in such plasmas [2].

Experiments on photoionization of ions face specific challenges associated with the need to match sufficiently dense ion beams with high-flux photon sources at short wavelengths [3]. Data on resonance structures and inner-shell thresholds for a wide variety of ions have been obtained through the dual laser-plasma photoabsorption technique

\footnotetext{
*Corresponding author. Electronic address: jeanpaul.mosnier@dcu.ie

${ }^{\dagger}$ Permanent address: GIK Institute of Engineering Sciences and Technology, Topi 23640, NWFP, Pakistan.

ॠElectronic address: jean-marc.bizau@lixam.u-psud.fr
}

PACS number(s): 32.80.Fb, 32.80.Dz

(DLP) which utilizes a laser-plasma ion source (see, e.g., the recent review by West [4]). While the DLP technique is extremely versatile in its application, DLP spectra can sometimes exhibit distortions due to the plasma environment in which case certain assumptions must be made in order to compare the measured relative cross-section data with theoretical calculations [5]. Furthermore, DLP experiments do not allow absolute cross sections to be determined. An alternative approach is to carry out photoion spectrometry on combined ion and undulator photon beams at a synchrotronradiation facility, the so-called merged beam technique. Such experiments provide excellent ion selectivity, do not suffer from the saturation effects present in photoabsorption, and most importantly, absolute cross sections can be determined. First pioneered in the 1980s [6], merged beam systems have recently been installed at several facilities including ASTRID (Aarhus), LURE (Orsay), Photon Factory (Tsukuba), and ALS (Berkeley).

The present paper describes absolute photoionization measurements on $\mathrm{Si}^{2+}$ ions (magnesium isoelectronic sequence) carried out at the SuperACO storage ring at LURE, Orsay. Aluminum and silicon are amongst the most abundant cosmic elements and information on their spectra in both neutral and ionic forms is necessary for the determination of stellar abundances, opacities, electron densities, etc., [7]. Our measurements were carried out in the photon energy ranges 
corresponding to the excitation or the ionization of a $2 p$-subshell electron. $2 p$ photoabsorption of singly and doubly ionized aluminum and silicon has been studied extensively using the DLP technique [8-12]. These data have provided insight into the changing nature of the resonant part of the cross section along the sequence; many assignments were also given using multiconfiguration atomic structure calculations. Most recently West and co-workers have used the merged beam facility at Aarhus to provide the corresponding $2 p$ photoexcitation/ionization cross-section data on an absolute scale for singly charged aluminum ions [13]. These authors also provided relabeling of a small number of resonances on the basis of their multiconfiguration Hartree-Fock calculations.

The present paper reports corresponding data for the isoelectronic partner $\mathrm{Si}^{2+}$. This data is compared with the results of advanced photoionization cross-section theoretical calculations, namely, the relativistic random-phase approximation (RRPA) and the relativistic multichannel quantumdefect theory (RMQDT). Thus, an insight into the $2 p$ photoionization behavior along the magnesium sequence is made possible. It has been shown recently that such a behavior may strongly depart from the increasingly hydrogenic behavior expected along an isoelectronic sequence [14].

\section{EXPERIMENTAL DETAILS AND RESULTS}

Detailed descriptions of the experimental setup and procedures used in our merged beam measurements can be found in Ref. [15]. In the present study, the silicon ions were produced, from silane, in an electron cyclotron resonance (ECR) ion source via plasma heating by a $10-\mathrm{GHz}$ microwave power supply. The ions were extracted by a highvoltage bias $(7 \mathrm{kV})$ and doubly charged $\mathrm{Si}^{2+}$ ions were selected by means of a Wien filter. Typical currents of $30 \mathrm{nA}$ of $\mathrm{Si}^{2+}$ were available in the interaction volume. The kinetic energy of the selected ion beam was lowered (by about $3 \mathrm{kV}$ ) in order to reduce collisional processes with the residual background gas, and to increase the density of the ion beam, in the region of overlap with the synchrotron photon beam. The doubly charged ion beam was aligned with the incoming photon beam using an electrostatic quadrupole deflector. The sideways and directional alignments of the two beams, termed the beam overlap, were measured by scanning wires across the beams at three locations placed before, after, and midway along the $0.20-\mathrm{m}$-long interaction region. The photoionization products were separated and analyzed using a cylindrical electrostatic deflector and a multichannel plate array detector.

The photon beam was produced by the undulator on the SuperACO SU6 beam line which is equipped with a 1-m toroidal grating monochromator and provides flux levels, covering the $30-180-\mathrm{eV}$ spectral range, of the order of $10^{12}$ (photons/s) $/ 0.1 \%$ BP at the exit slit. The $3 d^{9} 5 p$ and $4 d^{9} 6 p$ resonances in $\mathrm{Kr}$ and $\mathrm{Xe}$, respectively, provided the photon energy calibration. All the photon energies quoted in the present study were corrected for the Doppler shift and carry an error of $\pm 0.1 \mathrm{eV}$. A chopper placed between the monochromator and the interaction region allowed for a sequential recording of the ion signal, with and without photons, thus allowing a correction for background collisional effects. The experimental photon energy resolution was determined by a compromise between ion count rate and the monochromator bandpass. In the region 110-130 eV (resonance region) this resolution was of the order of $0.3 \mathrm{eV}$, whereas above $135 \mathrm{eV}$ (continuum region) a value of about $1 \mathrm{eV}$ was used. Both the single-photoionization (to $\mathrm{Si}^{3+}$ ) and double-ionization (to $\mathrm{Si}^{4+}$ ) cross sections have been determined. Cross-section values were obtained by normalizing the count rates for all the data points to the count rates of the points at which absolute measurements were carried out. The absolute cross sections obtained were $(6.45 \pm 0.85) \mathrm{Mb}$ at $133.2 \mathrm{eV}$ of photon energy and (4.34 \pm 1.2$) \mathrm{Mb}$ at $136.2 \mathrm{eV}$ of photon energy in the single- and double-photoionization channels, respectively.

The following formula was used to convert the count rate $S$ of triply or quadruply charged ions to cross section values $\sigma$ :

$$
\sigma=\frac{S Q v}{(I / \eta e)(J / e) \epsilon T F}
$$

In this formula, $Q$ is the charge of the target ions; $e$ the electron charge; $v$ the velocity of the ions in $\mathrm{m} / \mathrm{s}$; $I$ the current produced by the photons on the calibrated photodiode (in A); $\eta$ the efficiency of the photodiode at the photon en$\operatorname{ergy} \mathrm{h} \nu$ (in $\mathrm{A} / \mathrm{W}) ; J$ the current of incident ions in the Faraday cup (in A); $\epsilon$ the efficiency of the microchannel plates; $T$ is the transmission of the two analyzers; and $F$ is the form factor in $\mathrm{m}^{-1}$. See Ref. [15] for details on how $\epsilon, T$, and $F$ are evaluated and further experimental details. All our cross section measurements are presented in Fig. 1. The total photoionization cross section would be obtained by summing the single- and double-photoionization cross-section data curves.

A comparison of Fig. 1 with the photoabsorption data of Ref. [9] shows that the weak resonances measured around 95-eV photon energy are due to the single photoionization of $\mathrm{Si}^{2+}$ ions in the metastable state $2 p^{6} 3 s 3 p^{3} P$. The measured cross sections of Fig. 1 are thus apparent cross sections comprising the contributions from both ground-state and metastable $\mathrm{Si}^{2+}$ atoms. For the purpose of quantifying the actual contribution of the metastable ions, we scanned the region around $95 \mathrm{eV}$ with improved counting statistics, yielding cross sectional data with a large signal-to-noise ratio. This data is displayed in Fig. 2(a) with the corresponding statistical error bars. We also used the optical oscillator strengths calculated in Ref. [9] to generate synthetic cross sections as a function of photon energy in Fig. 2(b). All the lines of the $2 p^{6} 3 s 3 p^{3} P \rightarrow\left(2 p^{5} 3 s^{2} 3 p\right)^{3,1} L$ transition array were included and given a Gaussian profile with a full width at half-maximum (FWHM) equal to $0.30 \mathrm{eV}$ (i.e. roughly equal to the experimental photon energy resolution). The good agreement between the two profiles of Figs. 2(a,b) justifies the use of the oscillator strengths calculated in Ref. [9] to estimate the metastable fraction in the present experiment. Thus, by comparing the measured intensity of suitable resonances with the corresponding oscillator strengths, the frac- 


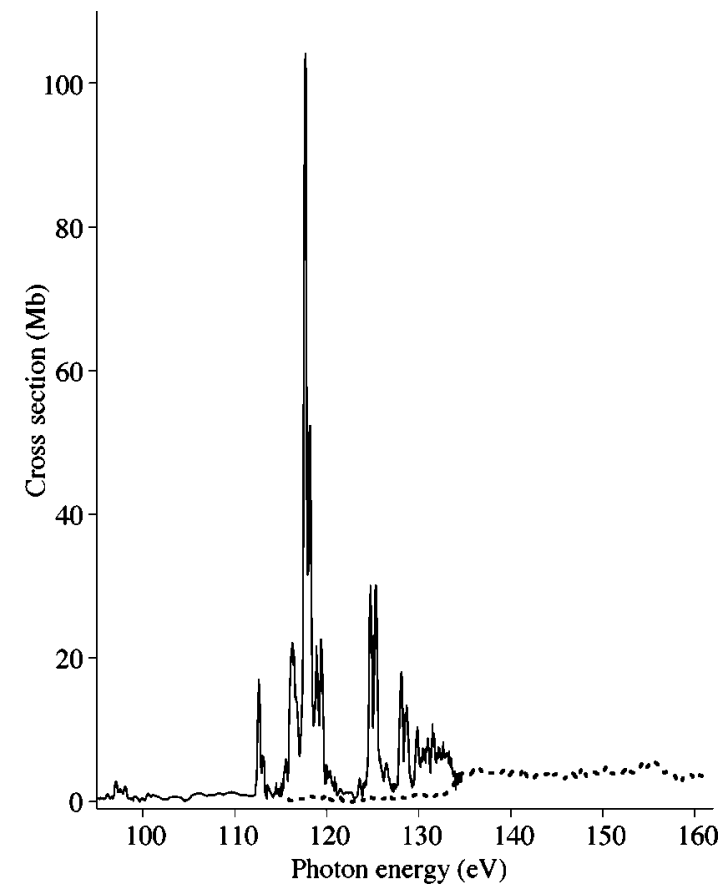

FIG. 1. Partial cross section of a $\mathrm{Si}^{2+}$ ion beam measured between $95 \mathrm{eV}$ and $135 \mathrm{eV}$ for the production of $\mathrm{Si}^{3+}$ (full line) and between $115 \mathrm{eV}$ and $165 \mathrm{eV}$ for the production of $\mathrm{Si}^{4+}$ (dashed line). The $\mathrm{Si}^{2+}$ ground-state ion beam contains $\sim 3 \%$ contaminant fraction of ions in the $2 p^{6} 3 s 3 p^{3} P$ metastable state (see text for details).

tion of $\mathrm{Si}^{2+}$ ions initially in the $2 p^{6} 3 s 3 p^{3} P$ metastable state can be estimated. Due to the smearing of the metastable resonances as a result of the selected photon energy resolution, we have broken down the metastable resonances into two distinct groups for which integrated intensities (after continuum subtraction) can be obtained accurately. The first one is centered around $96.0 \mathrm{eV}$ and the second one extends roughly between $97.0 \mathrm{eV}$ and $98.5 \mathrm{eV}$. The corresponding

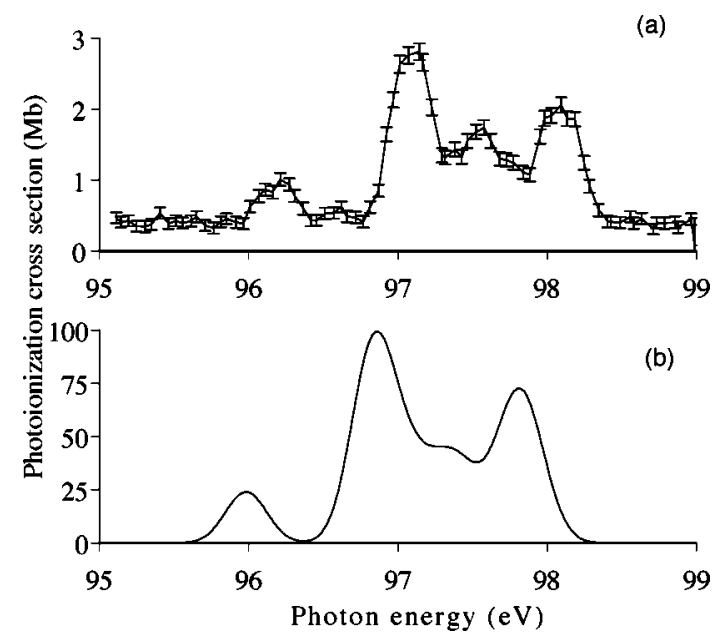

FIG. 2. (a) Resonance structure in the partial cross section for the production of $\mathrm{Si}^{3+}$ from $2 p^{6} 3 s 3 p^{3} P$ metastable $\mathrm{Si}^{2+}$ ions measured in the present experiment and (b) simulated using the multiconfigurational atomic structure calculations from Ref. [9] assuming a Gaussian profile of width $0.30 \mathrm{eV}$ for all the resonances. oscillator strength values are appropriately summed for both groups. By forming appropriate ratios between the measured intensities and the calculated ones, we obtained a metastable fraction $x$ such that $2 \% \leqslant x \leqslant 3 \%$.

\section{CALCULATIONS}

$A b$ initio calculations of the photoionization of $\mathrm{Si}^{2+}$ were performed using the RRPA methodology $[16,17]$. The calculation included all single excitations from the $3 s, 2 p, 2 s$, and $1 s$ subshells, i.e., the following 11 relativistic channels were considered: $3 s \rightarrow k p_{3 / 2,1 / 2} ; 2 p_{3 / 2} \rightarrow k d_{5 / 2,3 / 2}, k s ; 2 p_{1 / 2}$ $\rightarrow k d_{3 / 2}, k s ; 2 s \rightarrow k p_{3 / 2,1 / 2}$; and $1 s \rightarrow k p_{3 / 2,1 / 2}$. where $k$ refers to both discrete excitations $(k=n)$ and ionizing transitions $(k=\epsilon)$. Correlation in the form of interchannel coupling among the 11 excitation channels is included in the RRPA methodology, along with the most consequential aspects of ground-state correlation [16,17]. Thus, the RRPA calculations include significant, but not all, correlations of importance. In particular, two-electron excitations are omitted.

In addition, it is difficult and inefficient to use the RRPA directly to obtain the photoionization cross section in the vicinity of the Rydberg autoionizing states. Instead, RMQDT is employed [18], using RRPA to provide the input parameters for the RMQDT calculation.

\section{ANALYSIS AND COMPARISON WITH THEORY}

\section{A. Single-photoionization cross section between $110 \mathrm{eV}$ and $135 \mathrm{eV}$ (production of $\mathrm{Si}^{3+}$ ions)}

We have summarized the relevant energy-level structure of the $\mathrm{Si}^{2+}$ ion in Fig. 3. The inner-shell levels are taken from Refs. $[9,10]$. The metastable level $2 p^{6} 3 s 3 p^{3} P$ is also shown. It is seen that the photon energy range $(90-165 \mathrm{eV})$ of the present study always exceeds the $2 p^{6}$ doubleionization threshold. The $2 p$ inner threshold was measured at $133.8(1) \mathrm{eV}$ in good accord with the values of $133.32 \mathrm{eV}$ and 133.84 for $2 p^{2} P_{3 / 2,1 / 2}$ obtained by Sayyad et al. [10].

Higher-excited inner-shell levels based on electron configurations such as $\left(2 p^{5} 3 s 3 p\right) n p$ or $\left(2 p^{5} 3 s 3 d\right) n s, n d$, although not observed in photoabsorption experiments [10], have been predicted to lie above the $\left(2 p^{5} 3 s^{2}\right)^{2} P_{3 / 2,1 / 2}$ thresholds [19]. These levels are only accessible from the ground state via processes involving the simultaneous excitation of more than one electron upon the absorption of a single photon. Other levels belonging to configurations of the type $\left(2 p^{5} 3 s 3 p\right) n d(n \geqslant 4)$ are also expected to lie above the $2 p$ limits and are directly accessible from the metastable $2 p^{6} 3 s 3 p^{3} P$ state.

The single-photoionization cross section between $110-\mathrm{eV}$ and $135-\mathrm{eV}$ photon energies are shown in the upper part of Fig. 4 with statistical error bars. We have not corrected these cross sections in view of the very small metastable fraction. The cross section values decreased rapidly at higher photon energies and no signal above noise levels could be detected above $135 \mathrm{eV}$. This spectrum is dominated by a strong pattern of discrete structures. These constitute the resonant part of the $3 s$ partial cross section and are due to the excitation of 


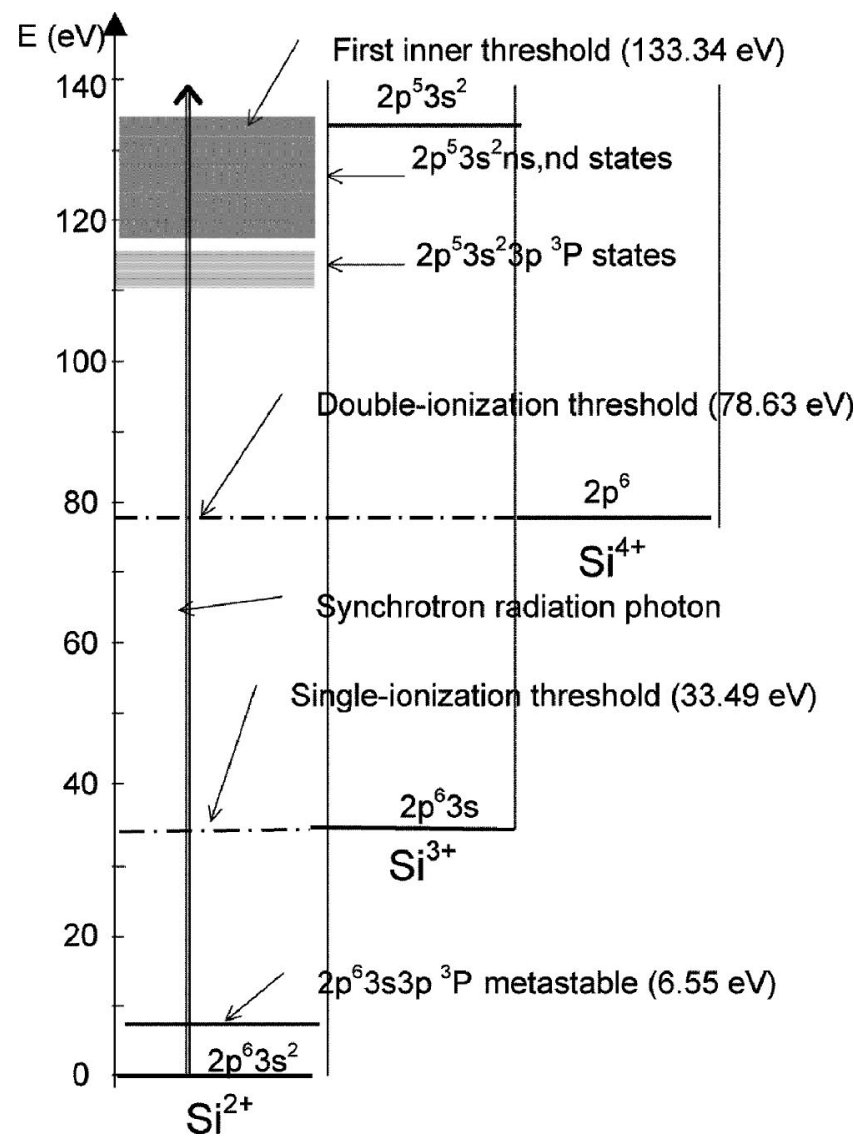

FIG. 3. Energy-level diagram of the $\mathrm{Si}^{2+}$ ion showing schematically the inner-shell resonances and thresholds relevant to the present study.

a $2 p$ electron into allowed core-excited levels. The contribution of the direct $3 s$ ionization process is, as expected, very small as can be judged by the strength of the corresponding underlying continuum. Our RRPA calculations give an average value of around $0.2 \mathrm{Mb}$ for the $3 s$ cross section in the interval $110-135 \mathrm{eV}$ while it has decreased to around $0.1 \mathrm{Mb}$ at $150 \mathrm{eV}$ (i.e., well above the $2 p$ threshold). Thus, above $133.8 \mathrm{eV}, 3 s$ photoionization is negligible and $2 p$ photoionization dominates the cross section (see the following section). This is consistent with the values of $\sigma_{2 p}=5.9 \mathrm{Mb}$ and $\sigma_{3 s}=0.09 \mathrm{Mb}$ in isoelectronic $\mathrm{Mg}$ measured at $80-\mathrm{eV}$ photon energy (about $20 \mathrm{eV}$ above $2 p$ threshold) in Ref. [20]. However, we note that this is in sharp contrast with the corresponding cross-section behavior in $\mathrm{Al}^{+}$near and above the $2 p$ threshold (at $91.71 \mathrm{eV}$ ) reported recently by West et al. [13].

These authors observed instead a sharp rise of $\sigma_{3 s}$ to values slightly above $1.0 \mathrm{Mb}$ (see their Fig. 1). Furthermore, it remained close to this value between $91.71-\mathrm{eV}$ and $155-\mathrm{eV}$ photon energies, with the $\sigma_{3 s} / \sigma_{2 p}$ ratio remaining close to $1 / 3$ throughout this range. At $100-\mathrm{eV}$ photon energy, for example, a value of around $0.2 \mathrm{Mb}$ is predicted for the $\mathrm{Al}^{+} 3 \mathrm{~s}$ cross section by the OPACITY calculations [21]. This body of theoretical and experimental evidence suggests that $\sigma_{3 s}$ measured by Ref. [13] above the $\mathrm{Al}^{+} 2 p$ threshold appears too large by a factor of $\sim 5$.

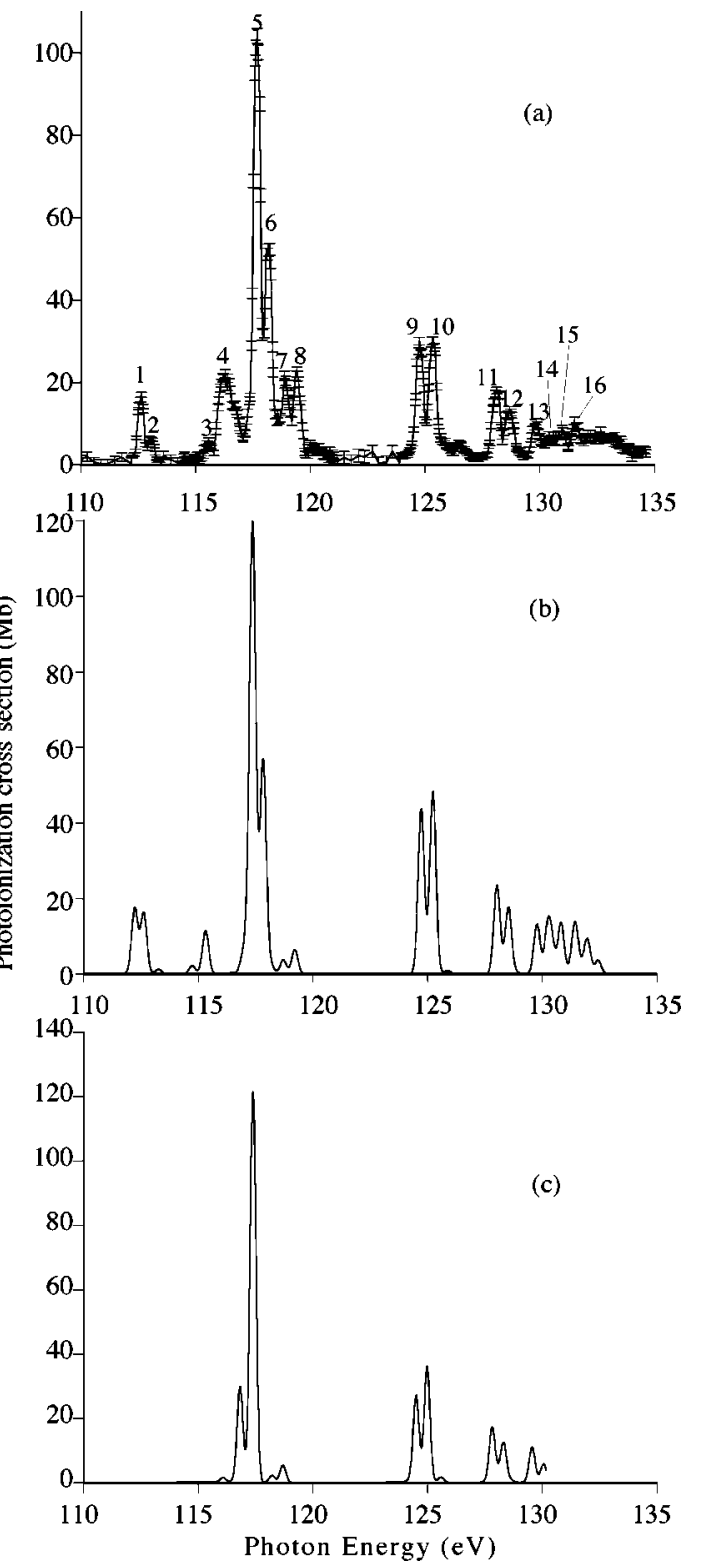

FIG. 4. Resonant structure in the $\mathrm{Si}^{2+}$ single-photoionization cross section in the photon energy range 110-135 eV: (a) experimental data (with statistical error bars) obtained by merging a $\mathrm{Si}^{2+}$ ion beam from an ECR source with monochromatized synchrotron radiation from an undulator and (b) synthetic cross section obtained by giving a Gaussian profile of FWHM $0.30 \mathrm{eV}$ to the oscillator strengths of the $2 p^{6} 3 s^{2} \rightarrow 2 p^{5} 3 s^{2} n s, n d$ resonances obtained in a multiconfigurational atomic structure calculation by Sayyad et al. [10], and (c) our RRPA-RMQDT calculations convolved with a Gaussian function of FWHM $=0.30 \mathrm{eV}$.

The dominant features of Fig. 4 are immediately identified as the $2 p^{6} 3 s^{2} \rightarrow\left(2 p^{5} 3 s^{2} n d\right)$ resonances with $n=3,4$, 5,6 , and 7 , respectively, while the other low-energy structures are due to excitations of states based on the $2 p^{5} 3 s 3 p^{2}$ and $2 p^{5} 3 s^{2} 4 s$ configurations, respectively. We have summarized all the data in Table I which compares the resonance energy positions between the photoion and the photoabsorption experiments. The resonance energies measured in the present experiment are about $0.2 \mathrm{eV}$ higher than the energies 
TABLE I. Measured energies, assignments, Rydberg analyses of the resonances measured in the single-photoionization cross section of $\mathrm{Si}^{2+}$ in the photon energy range $110-135 \mathrm{eV}$, and comparison with the photoabsorption data from Ref. [10].

\begin{tabular}{|c|c|c|c|c|c|}
\hline $\begin{array}{l}\text { Resonance } \\
\text { number } \\
\text { (Fig. 4) }\end{array}$ & Assignment & $\begin{array}{c}\text { Energy }(\mathrm{eV}) \\
\text { Present } \\
{ }^{\mathrm{c}} \\
\text { experiment }\end{array}$ & $\begin{array}{c}\text { Energy }(\mathrm{eV}) \\
\text { Photoabsorption data } \\
\text { from Ref. [10] }\end{array}$ & $\begin{array}{c}n^{* \mathrm{a}, \mathrm{b}} \\
{ }^{2} P_{3 / 2} \text { limit }\end{array}$ & $\begin{array}{c}n^{* \mathrm{a}, \mathrm{b}} \\
{ }^{2} P_{1 / 2} \text { limit }\end{array}$ \\
\hline 1 & $2 p^{5} 3 s\left({ }^{3} P\right) 3 p^{2}\left({ }^{1} D\right){ }^{3} D_{1}$ & 112.5 & 112.30 & & \\
\hline 2 & $2 p^{5} 3 s\left({ }^{1} P\right) 3 p^{2}\left({ }^{1} D\right){ }^{1} P_{1}$ & 112.9 & 112.80 & & \\
\hline 3 & & 115.5 & Not observed & & \\
\hline 4 & $2 p^{5} 3 s\left({ }^{3} P\right) 3 p^{2}\left({ }^{1} S\right){ }^{1} P_{1}+3 p^{2}\left({ }^{3} P\right){ }^{3} S_{1}$ & 116.2 & $(115.81+116.06)$ & & \\
\hline 5 & $2 p^{5} 3 s^{2}\left({ }^{2} P\right) 3 d^{1} P_{1}$ & 117.6 & 117.34 & $2.80(2.76)$ & \\
\hline 6 & $2 p^{5} 3 s^{2}\left({ }^{2} P\right) 3 d^{3} D_{1}$ & 118.1 & 117.88 & & $2.80(2.77)$ \\
\hline 7 & $2 p^{5} 3 s^{2}\left({ }^{2} P\right) 4 s^{3} P_{1}$ & 118.8 & 118.63 & & \\
\hline 8 & $2 p^{5} 3 s\left({ }^{3} P\right) 3 p^{2}\left({ }^{3} P\right){ }^{1} P_{1}$ & 119.3 & 119.12 & & \\
\hline 9 & $4 d[3 / 2,5 / 2]_{1}$ & 124.6 & 124.47 & $3.78(3.72)$ & \\
\hline 10 & $4 d[1 / 2,3 / 2]_{1}$ & 125.3 & 125.02 & & $3.78(3.72)$ \\
\hline 11 & $5 d[3 / 2,5 / 2]_{1}$ & 128.0 & 127.78 & $4.85(4.70)$ & \\
\hline 12 & $5 d[1 / 2,3 / 2]_{1}$ & 128.6 & 128.35 & & $4.88(4.72)$ \\
\hline 13 & $6 d[3 / 2,5 / 2]_{1}$ & 129.7 & 129.53 & $5.93(5.68)$ & \\
\hline 14 & $6 d[1 / 2,3 / 2]_{1}$ & 130.3 & 130.10 & & $5.99(5.72)$ \\
\hline 15 & $7 d[3 / 2,5 / 2]_{1}$ & 130.9 & 130.62 & $7.23(6.73)$ & \\
\hline 16 & $7 d[1 / 2,3 / 2]_{1}$ & 131.4 & 131.24 & & $7.25(6.86)$ \\
\hline
\end{tabular}

${ }^{\mathrm{a} 2} P_{3 / 2,1 / 2}$ thresholds at $133.32 \mathrm{eV}$ and $133.84 \mathrm{eV}$, respectively.

${ }^{\mathrm{b}}$ The number in brackets is the $n^{*}$ value obtained using the photoabsorption data.

${ }^{\mathrm{c}}$ Error $0.1 \mathrm{eV}$.

${ }^{\mathrm{d}}$ Error $0.05 \mathrm{eV}$.

measured in photoabsorption. For most of the resonances, these two sets of energies are in reasonable agreement taking into account their respective error bars. We follow the assignments given in Ref. [10] for the resonances numbered $1-8$ in Table I. Note that these assignments are given as the leading term in a multiconfiguration Hartree-Fock (MCHF) expansion of the wave function in $L S J$ coupling. We note that in Ref. [10] where all the resonances were labeled in the LSJ coupling scheme, some inconsistencies are present in the overall term assignments for the $4 d$ and $5 d$ resonances. For those higher-lying resonances, $j-j$ coupling is more appropriate [19] and all the resonances $2 p^{5} 3 s^{2} n d$ with $n \geqslant 4$ (numbered 9-16 in Table I) were relabeled accordingly. This is also consistent with the labeling scheme provided in Ref. [13] for the corresponding resonances in $\mathrm{Al}^{+}$and the Rydberg analysis shown in Table I. This quantum-defect analysis is based on the experimental thresholds measured in Ref. [10]. It shows distinct hydrogenic behavior and the absence of any significant perturbation of the $n d$ series $(n \geqslant 4)$ due to configuration mixing with the $n p^{2}$ doubly excited configurations. It is well known that such mixing dominates the cross section of magnesium in the $2 p$ excitation region (see references in Ref. [10]) and still plays an important role in $\mathrm{Al}^{+}$ [13]. These perturbations do have some effect upon the $2 p^{6} 3 s^{2} \rightarrow 2 p^{5} 3 s^{2} 3 d$ transition, as evidenced by the fact that, as seen in Table I, the ${ }^{1} P$ resonance lies below the ${ }^{3} D$ resonance, in contradistinction to Hund's rule.

As the data of Fig. 4(a) show a negligible underlying continuum, we have used the optical oscillator strengths obtained in the MCHF calculations of Sayyad et al. [10] to simulate the cross section, which are presented in Fig. 4(b). The agreement between Figs. 4(a) and 4(b) appears very sat- isfactory with respect to the resonance energies, relative strengths, and absolute intensities. This indicates that radiative decay of the resonances is negligible and the photoabsorption cross section is closely represented by the singlephotoionization cross section in this photon range. The multiconfiguration expansion used in the calculations of Ref. [10] appears thus well suited to the description of $2 p$ coreexcited levels in $\mathrm{Si}^{2+}$.

We have also performed RRPA-RMQDT calculations of the $3 s$ photoionization cross section that includes $2 p_{1 / 2,3 / 2}$ $\rightarrow n s, n d_{3 / 2,5 / 2}$ resonances. The results of these calculations are presented in Fig. 4(c) that shows the convolved cross section as a function of photon energy. We emphasize that the RRPA calculations do not include double excitations. Thus, the resonances in the region of $112.5 \mathrm{eV}$ are not present in the RRPA result; these resonances are seen in Table I to be double excitations. Furthermore, in the 117-eV region, RRPA correctly predicts two prominent resonances. However, the relative intensities of these resonances are exactly opposite to our experimental results and the theoretical $\mathrm{MCHF}$ results. This too is a consequence of the omission of double excitations in the RRPA calculation. As seen in Table I, the ${ }^{1} P$ multiplet of the $\left(2 p^{5} 3 s^{2} 3 d\right)$ configuration lies $b e$ low ${ }^{3} D$, in opposition to Hund's rule, because of the mixing with doubly excited configurations. Thus, the largest intensity, which goes, of course, to the $L S$ allowed ${ }^{1} P$ multiplet, appears at the lower energy. The RRPA result, which does not include this mixing, finds the ${ }^{1} P$ multiplet higher in energy than ${ }^{3} D$, and (incorrectly) predicts the large intensity in the higher-energy member of the pair. For the higher $\left(2 p^{5} 3 s^{2} n d\right)$ excitations, the mixing is much less important [19]. Here then, RRPA results should be good, and the com- 
parison in Fig. 4 shows that indeed to be the case; the agreement between RRPA theory and experiment is reasonable for the $4 d$ excitations, and nearly perfect for $5 d$. This implies that mixing with the double excitations still has some effect for the $4 d$ excitations.

We have not attempted to systematically extract the discrete oscillator strengths from the absolute cross-section data as many of the resonance wings are smeared. Only the two groups of the $4 d$ (lines 9 and 10) and $5 d$ (lines 11 and 12) resonances appear sufficiently well isolated for any integrated intensity measurement to be reasonably accurate. We obtain values for the summed oscillator strengths of 0.31 \pm 0.04 ( $4 d$ lines) and $0.15 \pm 0.02$ ( $5 d$ lines), uncorrected for the metastable contribution; these should be compared with calculated values of 0.286 (i.e., $0.136+0.15$ ) and 0.126 $(0.041+0.032+0.053)$, respectively, [10]. Again, the agreement between the two sets of numbers is very satisfactory and confirms our previous conclusions regarding the reliability of the calculations.

Finally, we propose that the small and rapidly diminishing signal contributing to the single-photoionization cross section between $133.8 \mathrm{eV}$ (i.e., above $2 p$ threshold) and $\sim 135 \mathrm{eV}$ is due to the allowed autoionizing decay of the multiply excited configurations discussed at the beginning of this section. The unpublished calculations by Sayyad appear to support this assignment [19].

\section{B. Double-photoionization cross section between $115 \mathrm{eV}$ and $165 \mathrm{eV}$ (production of $\mathrm{Si}^{4+}$ ions)}

The measured double-photoionization cross section are presented with statistical error bars in Fig. 5(a) as a function of photon energy in the range $115-165 \mathrm{eV}$. A number of atomic processes can contribute to the production of $\mathrm{Si}^{4+}$ ions in this range (see Fig. 3). However, only one continuum process strongly dominates the cross section above $133.5 \mathrm{eV}$ : photoionization of a $2 p$ electron followed by the Auger decay of the $2 p$ hole. A strong autoionizing resonance with characteristic asymmetric Fano profile is superimposed at $158-\mathrm{eV}$ photon energy. This corresponds to the excitation of a $2 s$-subshell electron (see below). A small window-type resonance is also present around $142 \mathrm{eV}$. Similar window resonances were also observed in $\mathrm{Al}^{+}$[13]. These had been observed previously and assigned to autoionizing levels of the $2 p^{5} 3 s 3 p 4 p$ configuration in Ref. [10], and we follow this assignment here. In Fig. 5(b), we present the results of our RRPA calculations of the $\mathrm{Si}^{2+} 2 p$ partial photoionization cross section. The experimental thresholds of Ref. [10] are used in this calculation which also includes the $2 s_{1 / 2}$ $\rightarrow n p_{1 / 2,3 / 2}$ resonances. For the sake of comparing the various theoretical approaches, we have also included the corresponding Dirac-Slater results embodied in the analytical fit of Verner et al. [22]. Calculated $2 p$ threshold energies are used in the latter and the continuum part only is included.

It is clear from the comparison that the RRPA results mirror the experiment in the 130-170-eV region quite well, both qualitatively and quantitatively. For the continuous (nonresonant) portion of the cross section, the RRPA result agrees with experiment to within the experimental error bars. In

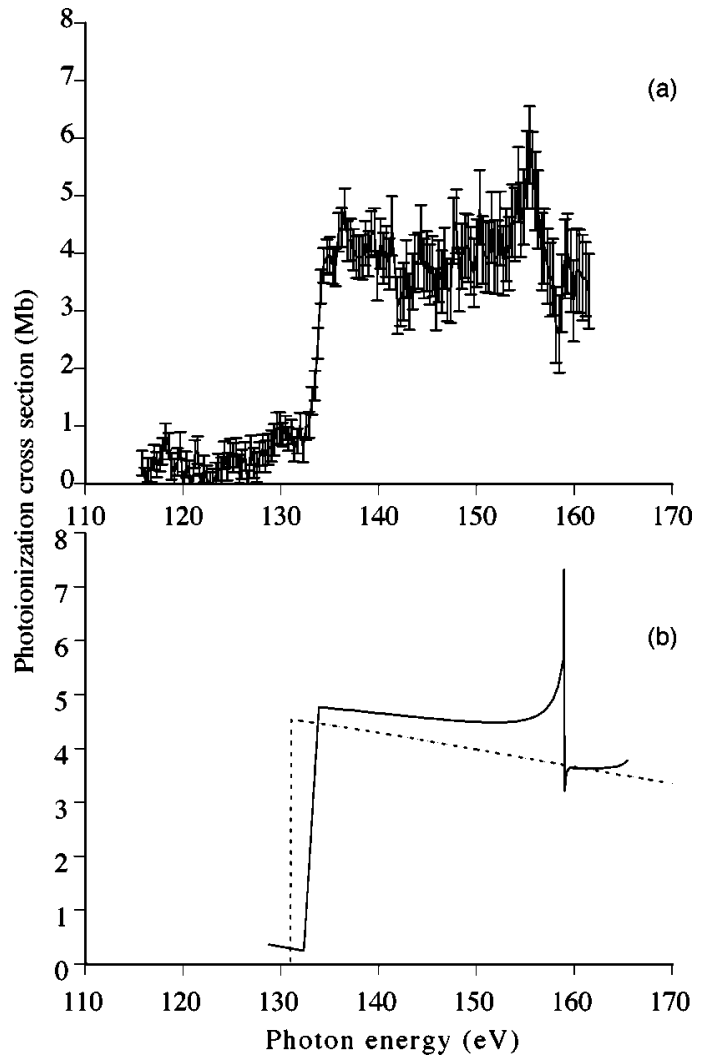

FIG. 5. $\mathrm{Si}^{2+}$ double-photoionization cross section in the region of the $2 p^{-1}$ threshold: (a) experimental data (with statistical error bars) in the range $115-165 \mathrm{eV}$ obtained by merging a $\mathrm{Si}^{2+}$ ion beam from an ECR source with monochromatized synchrotron radiation from an undulator and (b) our RRPA calculations (solid line), analytical fit to Dirac-Slater results of Verner et al. [22] (broken line)

addition, the $2 s \rightarrow 3 p_{1 / 2,3 / 2}$ (unresolvable) resonances appear just below $160 \mathrm{eV}$, but the theoretical position is slightly higher than the experimental. Note that the theoretical RRPA result has not been convolved with the experimental resolution, which is the reason that the theoretical resonance structure is so much higher and sharper than the experimental. Note also that the window resonance in the $142-\mathrm{eV}$ region is not predicted by RRPA since it results from a two-electron excitation. We could not detect any definite signals in the double-ionization channel at the photon energies corresponding to the intense resonances observed in the singleionization channel. This suggests that the contribution of the direct double-ionization process (threshold at $78.6 \mathrm{eV}$ ) is extremely small and not measurable in our experimental conditions. Similarly to Ref. [13], we observed enhancements in the cross section, just below threshold, in the interval 125$131 \mathrm{eV}$. It is the region where a photon can excite $2 p^{6} 3 s 3 p$ metastable $\mathrm{Si}^{2+}$ ions to levels belonging to the $2 p^{5}(3 s 3 p)$ nd configurations. $\mathrm{A} \mathrm{Si}^{4+}$ ion is then produced upon autoionization to $2 p^{5} 3 s^{2}$ which subsequently Auger decays to $2 p^{6}$ (resonant Auger process). Due to the similarity between the observed patterns in $\mathrm{Al}^{+}$and $\mathrm{Si}^{2+}$, we follow Ref. [13] and propose that this resonant Auger process originating in the metastable ions contributes to the cross-section 
enhancements in the double-ionization channel just below the $2 p$ threshold.

\section{CONCLUSIONS}

We have presented the absolute photoionization cross sections of doubly ionized silicon in the ground state as a function of photon energy in the region of the $2 p$ threshold ( 95 $\mathrm{eV}-170 \mathrm{eV})$. These were obtained using the merged beam experimental technique. Both the single- and double photoionization cross sections were measured. The cross sectional values contained a small contribution from metastable $\mathrm{Si}^{2+}$ that did not exceed $3 \%$.

The relativistic random-phase approximation (RRPA) was used to calculate the $2 p$ photoionization continuum cross sections and agreed very well with the corresponding measurements. An intense resonance structure was found to dominate the cross sections below the $2 p$ threshold. This structure was well reproduced by synthetic cross sections generated from optical oscillator strengths obtained in an $a b$ initio multiconfigurational atomic structure calculation and convolved with a Gaussian function to simulate the experimental resolution. On the other hand, RRPA-RMQDT calculations were less successful in reproducing this resonance structure and in particular, were unable to account for double excitations as such processes are not included in RRPA. From the experimental data presented, and the comparison with theory, it is clear that satellite transitions play an important role in the photoionization of $\mathrm{Si}^{2+}$. To treat this situation accurately requires going beyond current theoretical methodologies. In our judgment, the most promising one which could include multiconfiguration, multichannel, relativistic, inner-shell, and satellite transition effects is the multiconfiguration relativistic random-phase approximation [23]. This technique has been applied to double excitations from the valence shell. Work on an extension to include inner-shell excitations, such as those exhibited in the photoionization of $\mathrm{Si}^{2+}$, is a significant effort and is in progress.

To conclude, we point out that measurements of the type presented in this paper are now being actively developed at synchrotron radiation facilities world wide, allowing very accurate photoionization cross sections in multiply charged ions to be fairly routinely obtained, over a wide photon energy range, at very high resolution. The measurements are now of high enough quality to reliably challenge existing theoretical methodologies. The ongoing interplay between advanced experiments and theoretical developments will benefit the understanding of the interaction of ionizing photons with multiply charged ions and the modeling of laboratory and astrophysical plasmas.

\section{ACKNOWLEDGMENTS}

The support of NASA, NSF, and DST (India) is acknowledged. E.T.K. and J.P.M. acknowledge the financial help from the EU Access to Large Scale Facility Program and Enterprise Ireland.
[1] http://cdsweb.u-stras.fr/topbase.html; http://wwwphys.llnl.gov/Research/OPAL; http://www.usm. unimuenchen.de/people/ip/iron-project.html

[2] Spectroscopic Challenges of Photoionized Plasmas, edited by G. Ferland and D.W. Savin, ASP Conference Series Vol. 247 (Astronomical Society of the Pacific, San Francisco, 2001).

[3] F. J. Wuilleumier and J.B. West, in VUV and Soft X-Ray Photoionization, edited by U. Becker and D. A. Shirley (Plenum, New York, 1996).

[4] J.B. West, J. Phys. B 34, R45 (2001).

[5] C. McGuinness, M. Martins, Ph. Wernet, B.F. Sonntag, P. van Kampen, J.-P. Mosnier, E.T. Kennedy, and J.T. Costello, J. Phys. B 32, L583 (1999).

[6] I.C. Lyon, B. Peart, J.B. West, and K. Dolder, J. Phys. B 19, 4137 (1986).

[7] J.A. Cardelli, K.R. Sembach, and B.D. Savage, Astrophys. J. 440, 241 (1995); E.M. Sion, F.H. Cheng, K.S. long, P.R. Szkody, L. Gilliland, Min Huang, and I. Hubeny ibid. 439, 957 (1995).

[8] J. Brilly, E.T. Kennedy, and J.P. Mosnier, J. Phys. B 21, 3685 (1988).

[9] J.P. Mosnier, J.T. Costello, E.T. Kennedy, L. Kiernan, and M.H. Sayyad, Phys. Rev. A 49, 755 (1994).

[10] M.H. Sayyad, E.T. Kennedy, L. Kiernan, J.-P. Mosnier, and J.T. Costello, J. Phys. B 28, 1715 (1995).

[11] J.T. Costello, D. Evans, R.B. Hopkins, E.T. Kennedy, L. Kier- nan, M.W.D. Mansfield, J.P. Mosnier, M.H. Sayyad, and B.F. Sonntag, J. Phys. B 25, 5055 (1992).

[12] J.T. Costello, E.T. Kennedy, J.-P. Mosnier, and M.H. Sayyad, J. Phys. B 28, 677 (1998).

[13] J.B. West, T. Andersen, R.L. Brooks, F. Folkmann, H. Kjeldsen, and H. Knudsen, Phys. Rev. A 63, 052719 (2001).

[14] H.S. Chakraborty, A. Gray, J.T. Costello, P.C. Deshmukh, G.N. Haque, E.T. Kennedy, S.T. Manson, and J.P. Mosnier, Phys. Rev. Lett. 83, 2151 (1999).

[15] J.M. Bizau, E. Bouisset, C. Blancard, J.P. Champeaux, A. Compant La Fontaine, C. Couillaud, D. Cubaynes, D. Hitz, C. Vinsot, and F.J. Wuilleumier Nucl. Instrum. Methods Phys. Res. B 205, 290 (2003).

[16] W.R. Johnson and K.T. Cheng, Phys. Rev. A 20, 978 (1979).

[17] W.R. Johnson, C.D. Lin, K.T. Cheng, and C.M. Lee, Phys. Scr. 21, 409 (1980).

[18] C.M. Lee and W.R. Johnson, Phys. Rev. A 22, 979 (1980).

[19] M.H. Sayyad, Ph.D. thesis, Dublin City University, 1994 (unpublished).

[20] A. Hausmann, B. Kämmerling, H. Kossmann, and V. Schmidt, Phys. Rev. Lett. 61, 2669 (1988).

[21] K. Butler, C. Mendoza, and C.J. Zeippen, J. Phys. B 26, 4409 (1993).

[22] D.A. Verner, D.G. Yakovlev, I.M. Band, and M.B. Trzhaskovskaya, At. Data Nucl. Data Tables 55, 233 (1993).

[23] K.-N. Huang and W.R. Johnson, Phys. Rev. A 25, 634 (1982). 